\title{
Structure of the Power Spectral Density of Galactic Cosmic Ray Variation during 1953-2016
}

\author{
Pauli Väisänen, Ilya Usoskin and Kalevi Mursula \\ Space Climate Research Unit, University of Oulu, Finland \\ email: pauli.vaisanen@oulu.fi
}

\begin{abstract}
Fluxes of galactic cosmic rays (GCR) observed at 1 AU are modulated inside the heliosphere at different time scales. Here we study the properties of the power spectral density (PSD) of galactic cosmic ray variability using hourly data from 31 neutron monitors (NM) from 1953 to 2016 . We pay particular attention to the reliability of the used datasets and methods. We present the overall PSD and discuss different parts of the spectrum and the related periodicities. We find significant spectral peaks at the periods of 11 years, 1.75 years, 155 days, 27 days and 24 hours and the harmonics of the latter two peaks. We calculate a power law slope of $-1.79 \pm 0.13$ for the period range between 50 and 130 hours and a slope of $-1.34 \pm 0.17$ for the period range between 40 days and 3.4 years $(1000-30000 \mathrm{~h})$.
\end{abstract}

Keywords. Space Climate, Cosmic rays, Heliospheric modulation

\section{Introduction}

The flux of galactic cosmic rays in the heliosphere is modulated by solar activity and, especially, by the changes in the heliospheric magnetic field (HMF). Irregularities in the HMF scatter cosmic ray particles, which is seen as a reduced intensity of GCR observed at Earth. These changes are monitored by the NMs which measure the secondary nucleons generated by the interaction of GCR with the Earth's atmosphere.

A common way to study the temporal variability and possible periodicities of a measured quantity is to examine its power spectral density (PSD), which shows the amount of power distributed at different frequencies. In addition to spectral peaks generated by systematic periodicities, the PSD typically exhibits a continuous power law behaviour which can be associated with turbulence, self-organized criticality and/or stochastic processes.

In this study we compute the PSDs of NM data using multiple NM stations. These PSDs are then analysed for different periodicities and power law behaviour.

\section{Data, methods and parameters}

We use NM data from 31 globally distributed stations listed in Figure 1 spanning the years 1953-2016, which correspond to roughly six solar cycles. Principles of the NM as a cosmic ray detector can be found elsewhere (e.g., Moraal et al. 2000; Simpson 2000; Usoskin et al. 2005). The full list of NMs and their operation periods is available at http://cidas.isee.nagoya-u.ac.jp/WDCCR/datacoverage_num.html.

Data were mostly obtained through the IZMIRAN data site at http://cr0.izmiran . $\mathrm{ru} /$ common/links.htm, which contains full-length datasets of NM data for most stations, containing also data from closed stations. Outliers, discontinuities and GLE events were removed using a median filter and visually examining the data. The median NM 

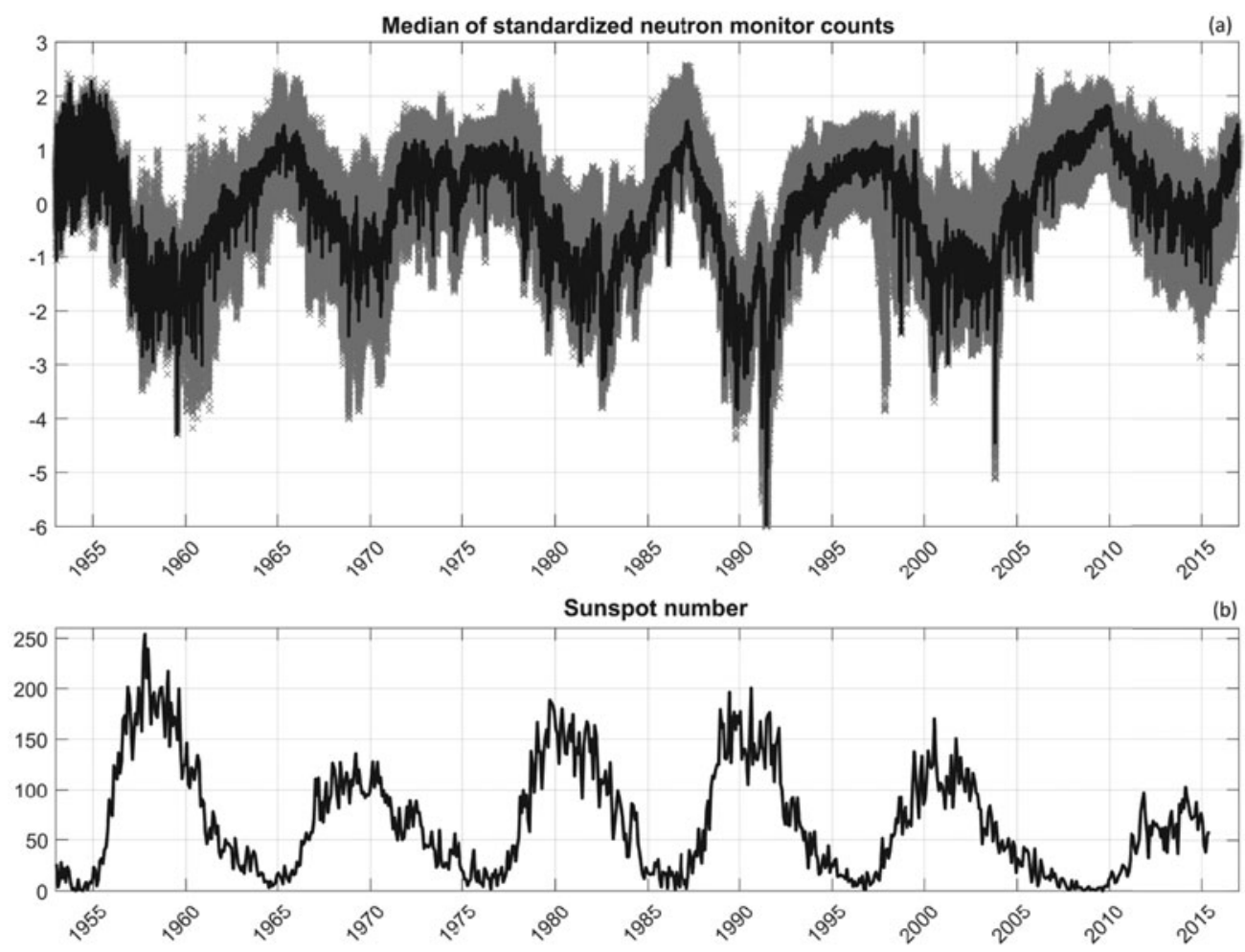

Amount of neutron monitors online during a 459 day window

(c)

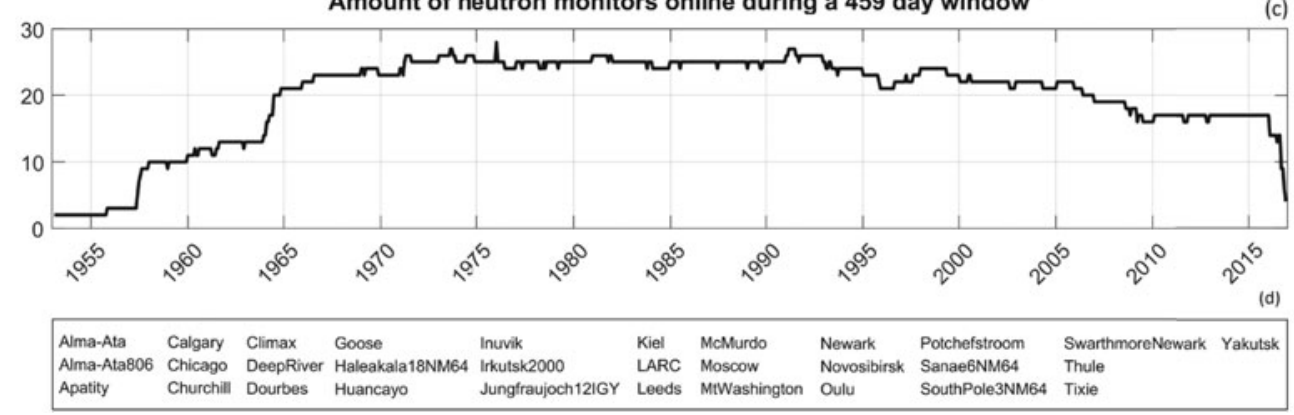

Figure 1. [a]: Median value (black line) of standardized count rates of 31 neutron monitors listed in panel [d]. [b]: Monthly sunspot number. [c]: Number of operating monitors during a 459-day (=17 solar rotations) time window.

count calculated from the standardized counts of all 31 NMs and the number of stations operating at each time are shown in Figure 1.

All the used datasets are linearly interpolated, detrended and standardized before calculating the PSD. For the PSD calculation we used the FFT with Welch method of averaging periodograms. This is a good PSD estimation method for data that includes data gaps (Munteanu et al. 2016). FFT transform of the signal is also computationally very fast. In the Welch method, the time series is first split into multiple, partly overlapping segments, and FFT and PSD are calculated for each segment. Then the segment PSDs are finally averaged to obtain a PSD with reduced noise. However, this process loses information at the low frequencies of the signal, depending on the used segment length. 


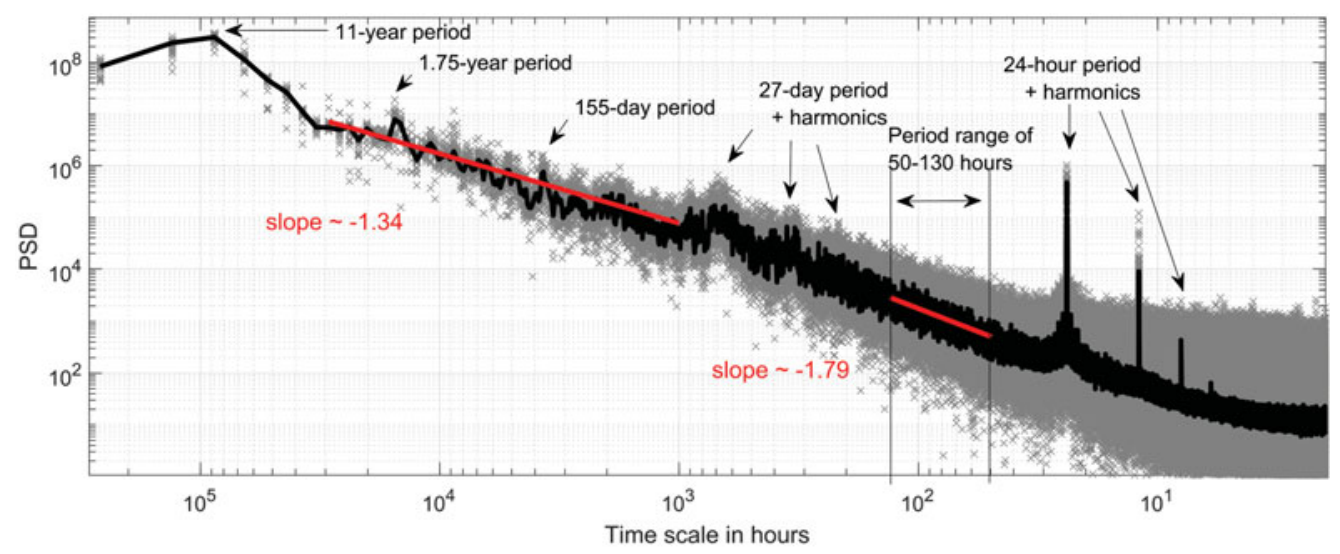

Figure 2. The median (black line) and individual monitor (gray points) power spectral densities calculated using the Welch method with segment length of $25 \%(\sim 16$ years $)$ and overlap of $95 \%$. Data from 29 NM stations was used. Slopes between 1000 to 30000 hours and between 50 to 130 hours are depicted in red.

In this study we calculate two PSDs using segment lengths of $25 \%$ and $1 \%$ of the total length of 63 years, which correspond to 16 years and 230 days of measurements, respectively. The longer segment length allows us to include the solar cycle periodicity in our PSD, and the shorter segment length allows us to study the higher frequency periodicities in more detail. For both cases the overlap of two successive segments is $95 \%$. For the $25 \%$ segment length we do not use a window function and for the $1 \%$ segment length we use a Hanning window.

\section{PSD analysis}

Figure 2 shows the median PSD and the PSDs of the individual stations calculated for the whole time series using the $25 \%$ segment length. Two stations (SwarthmoreNewark and Haleakala(18NM64)) had less than 16 years of measurements, so their data was not used. We calculated the PSD for all other NMs separately and took the median of the 29 PSDs at each frequency. The frequencies are transformed into periods for easier identification.

The highest PSD peak is observed at the 11-year mark, which corresponds to the solar cycle. The solar cycle is the most prominent driver of change in the space conditions (space climate), and many other solar periodicities are caused or influenced by it.

A midterm quasiperiodicity is observed at around 1.75 years, identified earlier in some other studies on cosmic ray data (Valdés-Galicia et al. 1996), as well as in solar wind speed and geomagnetic activity (Mursula et al. 2003).

The Rieger periodicity, which has been suggested to be connected to the Rossby waves in the tachocline (Zaqarashvili et al. 2010) is observed at around 155 days. A weak second harmonic at 77 days is also visible.

Another solar activity related peak is at the 27-day period of solar rotation (see, e.g., Gil and Mursula, 2017, and references therein). Differential rotation and the evolution of active regions and coronal holes on the solar surface cause the 27-day peak to be wide. The 13.5-day second harmonic of the solar rotation can also be clearly observed, and, less clearly, also the 9-day third harmonic. The diurnal variation, which is stronger than the solar rotation period, is seen at the 24-hour mark. The 2 nd and 3 rd harmonics at 12 and 8 hours are also clearly noticeable. 


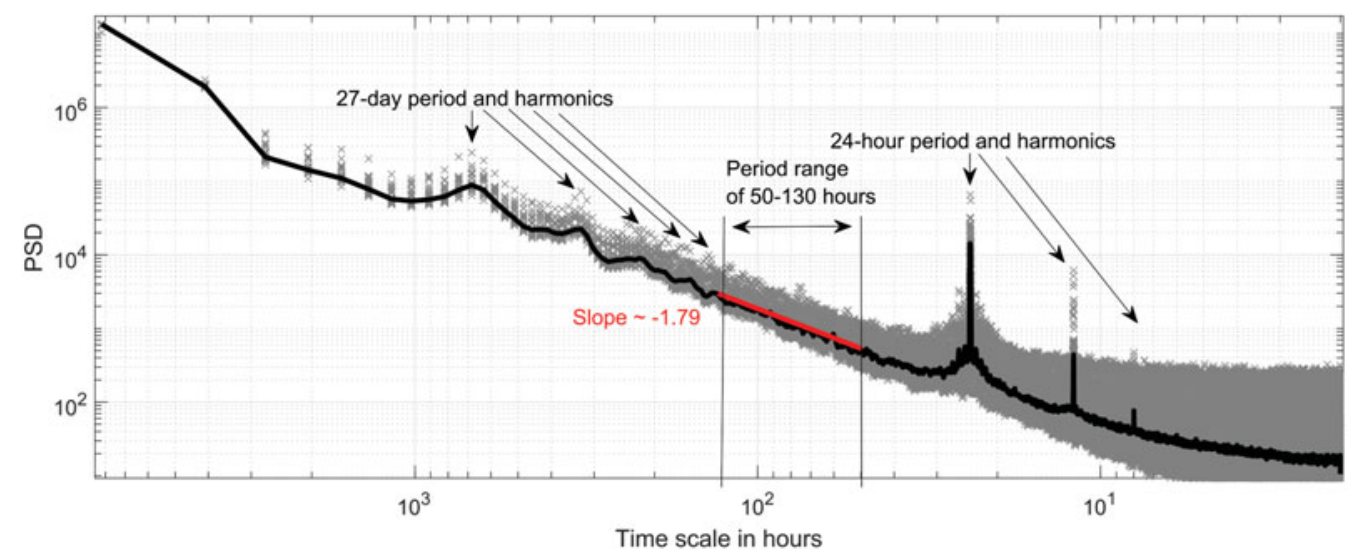

Figure 3. The median (black line) and the individual (gray points) power spectral densities calculated using the Welch method with the segment length of $1 \%$ ( 230 days), overlap of $95 \%$ and a Hanning window using all 31 NM stations. Slope of -1.79 (between 50 to 130 hours) is depicted in red.

All the identified main peaks are significant at the confidence level better than $95 \%$, determined by comparing the lower $95 \%$ confidence bound of the peak PSD value to the upper confidence bound value of points before and after the peak.

At time scales less than 10 hours we can observe the PSD flatten to white noise.

Figure 2 also outlines the time scales between 50 and 130 hours which exhibits a range of periods least affected by power peaks and a clear power-law behaviour. This part of the spectrum is between the harmonics of the 27-day peak and the diurnal peak. The power of this period was earlier studied, e.g., by Kudela et al. (1991). We calculate the PSD slope for each monitor and obtain a mean slope of -1.79 and the standard deviation of \pm 0.13 from the individual slopes. At the low frequency part of the spectrum between 1000 and 30000 hours we calculate a slope of -1.34 with a standard deviation of \pm 0.17 , which indicates a spectral break around the solar rotation period. This lower frequency part of the spectrum is, however, affected by spectral peaks.

Figure 3 gives a closer look at the high-frequency part of the power spectrum. Using a segment of $1 \%$ of the total time, we obtain an smoother spectrum than in Figure 2 . All the $31 \mathrm{NMs}$ are now used. The spectrum is quite smooth below the 200-hour mark, but small peaks at the fourth and fifth harmonics of solar rotation period at 164 and 132 hours are the reason why 130 hours is used here as the lower cut-off frequency for this part. At the higher frequency end of this part we observe an increase of the spectrum towards the diurnal peak, which sets the selected 50 hours as the higher frequency cutoff for this part. For this PSD between 50 and 130 hours we calculate a mean slope of $-1.79 \pm 014$.

\section{Conclusions}

We have calculated the power spectral densities of neutron monitor counts from 31 stations that span the years 1953-2016. Two power spectral densities were calculated, one covering all periods up to 16 years, the other for high frequencies above one year.

The strongest peaks in the power spectral densities are the 11-year, 1.75-year, 155-day, 27-day and 24-hour peaks. The second and third harmonics of 27-day and the 24-hour periodicities are also clearly present in the spectrum. The most clear power-law is at frequencies corresponding to time scales between 50 and 130 hours, where the slope was 
$-1.79 \pm 0.13$. At lower frequencies between 1000 and 30000 hours (about 40 days to 3.4 years) we observe a slope of around $-1.34 \pm 0.17$, which indicates a spectral break around the solar rotation period.

\section{Acknowledgements}

We acknowledge the financial support by the Academy of Finland to the ReSoLVE Centre of Excellence (project no. 272157).

\section{References}

Gil, A. \& Mursula, K. 2017, A\& A, 599, A112

Moraal, H., Belov A. \& Clem J. M. 2000, Space Sci. Revs, 93, 285-303

Munteanu, C., Negrea, C., Echim, M. \& Mursula, K. 2016, AG, 34, 437

Mursula, K., Zieger, B. \& Vilppola, J. H. 2003, Solar Phys., 7, 123

Kudela, K., Ananth, A. G. \& Venkatesan, D. 1991, J. Geophys. Res.: Space Physics, 96, 2156

Simpson, J. A., 2000, Space Sci. Revs, 93, 11-32

Usoskin, I. G., Alanko-Huotari, K., Kovaltsov, G. A. \& Mursula, K., 2005 J. Geophys. Res., 110, A12108

Valdés-Galicia, J. F., Pérez-Enríquez, R. \& Otaola, J. A. 1996, Solar Phys., 167, 409

Zaqarashvili, T. V., Carbonell, M., Oliver, R. \& Ballester J. L. 2010, ApJ, 709, 749 